\section{Unvermutete Polstermöbel-Allergie}

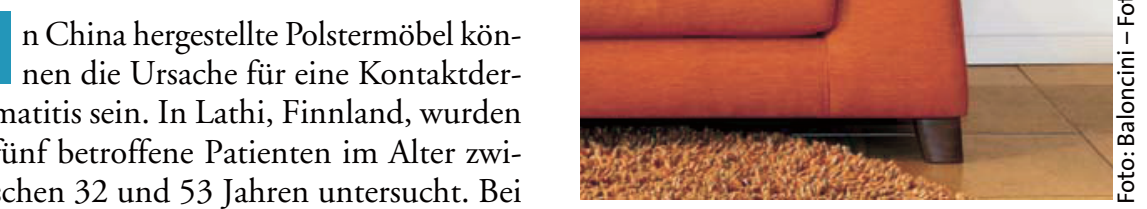
allen Patienten schlug eine Therapie der Hautläsionen mit Glukortikoiden zunächst nicht an, erst nach Meiden des Möbels heilten die Dermatitiden unter Lokaltherapie innerhalb von einigen Wochen ab. Die durchgeführten Patchtests, unter anderem mit Plastikmaterialien, Klebern und Textilfarben, blieben ohne Erfolg. Positive Reaktionen ergab eine von einer Patientin mitgebrachte Stoffprobe, aus der dann Dimethylfumarat (DMF) als auslösendes Agens ermittelt werden konnte. Positive Patchreaktionen wurden bei allen getesteten Patienten bei einer Konzentration von 0,001\% beobachtet, die empfindlichste Patientin reagierte noch auf $0,0001 \%$ DMF in wässriger Lösung. DMF wurde den chinesischen Stoffen wahrscheinlich aufgrund seiner antimikrobiellen und fungiziden $b k$

Rantanen T. Br J Dermatol 2008; 159: 218-21 Eigenschaften zugesetzt.

\section{England holt auf}

D isher war in Englands Hausarztpraxen die Anamnese das wichtigste und meist auch einzige Instrument der allergologischen Diagnostik. Ob ein zusätzlicher Pricktest die Trefferquote verbessern kann, wurde jetzt bei vier „general practioners" und mit 127 allergischen Probanden untersucht. Das Ergebnis mag auch manchen Kontinentaleuropäer noch beeindrucken: Ausschließlich auf die Selbstauskunft der Patienten gestützt, lag der Anteil falsch positiver Diagnosen zwischen $27 \%$ bei vermuteter Hundeallergie und $75 \%$ bei vermuteter Hausstaubmilbenallergie.

Smith HE et al. J Allergy Clin Immunol 2009; 123: 646-50

\section{Alternative gefragt}

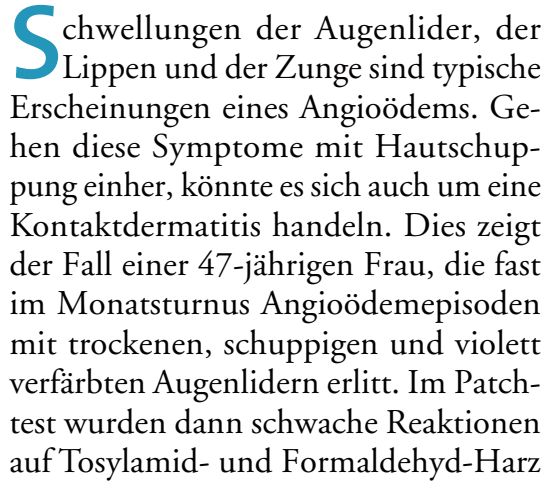

und eine stark positive Reaktion gegenüber dem Nagelhärter der Patientin nachgewiesen. Tosylamid und Formaldehyd sind häufige Bestandteile von Nagellack und Nagelhärtern. Mit diesem Befund konnten auch die Zungenschwellungen der Patientin erklärt werden, die stets nach dem Abkauen des Nagellacks auftraten.

$b k$

Moran B et al. Contact Dermatitis 2009;

6o: $114-5$

\title{
H1-Blocker bitte schlucken
}

sich eine schmerzhafte unregelmäßige Ulzeration an der Mundbodenschleimhaut sowie auf der gegenüberliegenden Seite unter der Zunge gebildet. Da die Frau Desloratadin sonst bei bestimmungsmäßiger Einnahme immer gut vertragen hatte, war die Ulzeration wohl nicht auf eine Überempfindlichkeit, sondern auf eine Verätzung zurückzuführen. Interessant an diesem Fall ist auch der Grund für die sublinguale Applikation: Die Frau erhoffte sich so eine stärkere schlafanstoßende Wirkung $b k$

Kluger N. J Eur Acad Dermatol Venereol 2009; 23: 234

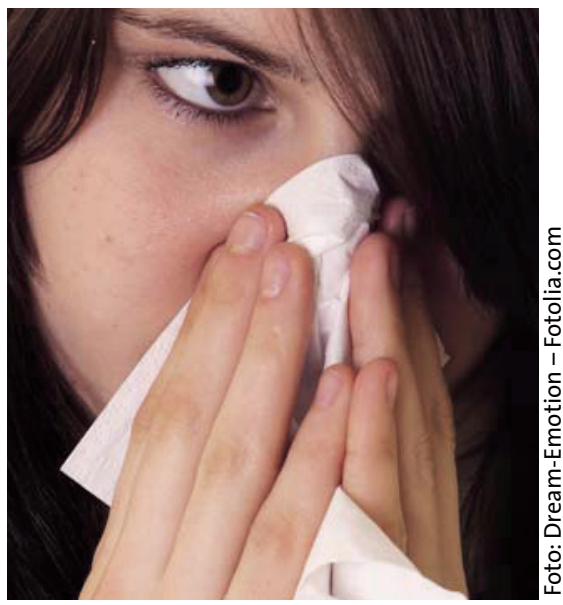

_ ine banale virale Rhinitis wird meist - begleitet von wunder Haut rund um die Nase. Tatsächlich zeigten biophysikalische Messungen an 14 Freiwilligen mit einer viralen Rhinitis, dass es während der Rhinitisepisode zu vermehrtem transdermalen Wasserverlust und Lipidbarrierestörungen der Hautoberfläche rund um die Nase kommt. Die Forscher führen ihre Beobachtungen auch auf den häufigen Gebrauch von Papiertaschentüchern zurück und fordern die Entwicklung innovativer Produkte.

Houben E et al. Contact Dermatitis 2008; 59: 296-300 